\title{
3. REZENSIONEN
}

\section{Britta Lange. 2013. Die Wiener Forschungen an Kriegsgefangenen 1915-1918: Anthropologische und ethnografische Verfahren im Lager. Wien: VÖAW.}

\author{
Gabriele Fröschl
}

Die zahlreichen Neuerscheinungen rund um das Gedenkjahr 100 Jahre Erster Weltkrieg 2014/1914 werden durch die vorliegende Publikation um einen interessanten Teilaspekt des Themenkomplexes ergänzt, indem die Verfasserin nicht das unmittelbare Kriegsgeschehen beleuchtet, sondern wissenschaftliche Forschung, die durch die Kriegssituation ermöglicht und begünstigt wurde. Im Zentrum der Arbeit, der ein vom FWF gefördertes Forschungsprojekt zugrunde liegt, stehen die Untersuchungen, die der Wiener Arzt und Anthropologe Rudolf Pöch gemeinsam mit anderen Wissenschaftskollegen zwischen 1915 und 1918 an mehr als 7.000 Kriegsgefangenen in Lagern der Habsburgermonarchie durchgeführt hat. Das Interesse an Forschung in den Kriegsgefangenenlagern ging von der Anthropologischen Gesellschaft aus, die in diesen Lagern ein ideales Forschungsumfeld sah. Einleitend beleuchtet Lange die Wissenschaftsgeschichte der Anthropologie und Ethnologie im 19. Jahrhundert und zeigt Parallelitäten zwischen Forschung im kolonialen Kontext und Forschung im Kriegsgefangenenlager. Pöch unternahm zu Beginn des 20. Jahrhunderts Forschungsreisen nach Westafrika, Australien, Neuguinea und Südafrika zum Zweck anthropologischer und ethnografischer Studien. Die Methodik dieser Feldforschung - vermessen, befragen, fotografieren, filmen, phonographieren - wurde bei seinen Forschungen in Kriegsgefangenenlagern fortgeführt - wobei im Lager der geografische, kulturelle und soziale Rahmen seiner früheren Feldforschungen nicht vorhanden war. Die vorliegende Arbeit zeigt sehr gut die Entwicklungen, die diese Wissenschaft im 19. und beginnenden Jahrhundert genommen hat, und wie Wissenschaftler im Rahmen ihrer Forschungen auf die herrschenden Strukturen der Kolonialverwaltung zurückgreifen konnten - Ähnlichkeiten mit der Situation in den Kriegsgefangenenlagern sind hier evident. 
In weiterer Folge widmet sich Lange drei Schwerpunkten: Vermessen und Fotografieren, Produktion von Filmen und der Herstellung von Tonaufzeichnungen; die Ergebnisse dieser Untersuchungen sind Substitute: Messbögen, Fotografien, Gipsabdrücke, Ton- und Filmaufnahmen.

Fotografie, Vermessungen und die Herstellung von Gipsabdrücken dienten der anthropologischen Visualisierung zur „Rassendiagnose“: Der wissenschaftlich geforderten „Objektivität" stand eine räumliche Inszenierung in Form eigener Räumlichkeiten für die wissenschaftlichen Untersuchungen zur Seite, die die naturwissenschaftlich medizinische Arbeitsweise unterstreichen sollten. Die Ermittlung von „Rassenmerkmalen“ geschah unter fragwürdigen Umständen (Nacktheit, fehlende Erklärung der Vorgangsweise) und diente vor allem zur Feststellung von statistischen Häufigkeiten und Modellen, die später auch als Basis für bevölkerungspolitische und rassenhygienische Fragestellungen herangezogen wurden. Neben der Vermessung erfolgte auch die fotografische Erfassung nach festgesetzten Schemata, die Ergebnisse wurden in Rassenmerkmale zergliedert. Zuletzt wurde unter dem Aspekt der "Objektivität“ ein Gipsabdruck des gesamten Kopfes erstellt. Einige dieser Exponate wurden bei der Kriegsausstellung im Sommer 1916 präsentiert.

Dies ist ein Beispiel für die Verbreitung der Forschung - spannend wäre auch gewesen, hier noch genauer zu untersuchen, wie und ob diese Forschung zeitgenössisch rezipiert wurde.

Die Filmaufnahmen aus den Kriegsgefangenenlagern Eger und Reichenberg widmen sich vor allem zwei Hauptthemenbereichen: handwerklichen Tätigkeiten und Tänzen/Ritualen, dazu kommt ein Film über das Abnehmen der Gipsabdrücke. Der Film ist hier eine wissenschaftliche Methode, eine Art von „Feldaufnahmen“ in der untypischen Umgebung des Lagers - wobei der Lageralltag völlig ausgeblendet bleibt. Diesen Filmen mit wissenschaftlichem Anspruch stehen zeitgenössisch die Propagandafilme des Ersten Weltkriegs gegenüber, die über diese Kriegsgefangenenlager berichten und ein Bild zeichnen, das das saubere und effektive Lager einer "Kulturnation“ den „exotischen“ Insassen gegenüberstellt. Ausgeblendet bleibt in der vorliegenden Arbeit weitgehend dieses System „Lager", die dahinterliegenden Strukturen und Mechanismen sowie der Alltag der zu Forschungsobjekten gemachten Insassen.

Die Stimmporträts wurden im Auftrag des Phonogrammarchivs erstellt, der Auftrag erlaubte eine breite inhaltliche Streuung (Sprache, Volkslied, Soldatenlied, Rekrutenlied). Die ersten Tonaufnahmen entstehen im Sommer 1915, auch mit dem Ziel, aussterbende Dialekte und Sprachen zu dokumentieren. Instrument dafür ist der am Phonogrammarchiv entwickelte Wiener Archiv-Phonograph, die Aufnahmen entstanden in einer Studiosituation, 
um beste technische Qualität zu gewährleisten. Neben der Aufnahme wurden Protokollbögen zur Erfassung der Person und Aufnahmesituation und Übertragungen der Aufnahmen in Schriftform (Transkriptionen) erstellt. Forschungsschwerpunkt waren vor allem die "Kaukasusvölker", die disziplinübergreifend (Sprachwissenschaft, Musikwissenschaft, Ethnologie, Geografie) beforscht wurden. Die Forscher waren im Lager weitgehend auf einheimische Übersetzer angewiesen, die auch den kulturellen Kontext herstellen konnten. Es bleibt anhand der zeitgenössischen Aufzeichnungen unklar, wer die Verfasser der gesprochenen Texte waren, die vorgetragen wurden, auch wenn man sie als freie Rede bezeichnete.

Nur wenige Aufnahmen beziehen sich unmittelbar auf Themen wie Krieg, Gefangenschaft, Sehnsucht nach der Heimat und drücken dabei auch Widerstand gegen die aktuelle Situation aus. Dass Teile dieser Aufnahmen bis heute unbearbeitet geblieben, inhaltlich nicht aufgearbeitet und entschlüsselt sind und nur im Archiv zugänglich, also bis heute ein archivalisches Sammelprojekt geblieben sind, mag aus Perspektive der heutigen Wissenschaftlerin bemerkenswert sein - entspricht aber archivalischer Realität. In diesem Zusammenhang von "gefangenen Stimmen“ zu sprechen, erscheint ein wenig zu negativ konnotiert.

Insgesamt ein lesenswertes Buch zu einer Thematik rund um den Ersten Weltkrieg, die bislang noch wenig im Fokus der Forschung gestanden ist.

Gabriele Fröschl: Historikerin, Leitung Österreichische Mediathek. 